Zdravko Kravanja (Editor), Proceedings of the $26^{\text {th }}$ European Symposium on Computer Aided Process Engineering - ESCAPE 26

June 12th -15th, 2016, Portorož, Slovenia (C) 2016 Elsevier B.V. All rights reserved.

\title{
An intelligent decision making and notification system based on a knowledge-enabled supervisory monitoring platform
}

\author{
Chrysovalantou Ziogou ${ }^{\mathrm{a}}$, Stelios Krinidis ${ }^{\mathrm{b}}$, Dimosthenis Ioannidis ${ }^{\mathrm{b}}$, Simira \\ Papadopoulou $^{\mathrm{c}}$, Dimitrios Tzovaras ${ }^{\mathrm{b}}$, Spyros Voutetakis $^{\mathrm{a}}$ \\ ${ }^{a}$ Centre for Research and Technology Hellas (CERTH), Chemical Process and Energy \\ Resources Institute (CPERI), 6th km Harilaou-Thermi rd, POBOX 60361, Thessaloniki, \\ 57001, Greece \\ ${ }^{b}$ Centre for Research and Technology Hellas (CERTH), Information Technologies \\ Institute (ITI), 6th km Harilaou-Thermi rd, POBOX 60361, Thessaloniki, 57001, Greece \\ ${ }^{c}$ Alexander Technological Educational Institute of Thessaloniki, Department of \\ Automation Engineering, PO Box 141, Thessaloniki, 54700, Greece
}

\begin{abstract}
This work describes a knowledge-based framework which is able to communicate online with process systems utilizing the existing infrastructure and provide knowledgeable actions to the operators. The proposed knowledge-enabled supervisory monitoring (KSM) platform combines components of existing standards such as ISA-95 in order to facilitate the data exchange between the automation system and the data repository. The functionalities of the developed platform are demonstrated using the requirements of an industrial control system of a continuous process and aim at the evaluation of the process and equipment performance based on predefined rules.
\end{abstract}

Keywords: decision-making, knowledge management, ontology engineering, information visualisation

\section{Introduction}

Continuous processes are complex systems that consist of various interconnected components that need to be monitored and controlled in an efficient way. The increasing level of automation that exists at these processes poses new challenges for the industry as the extraction is useful information and valuable knowledge is an important aspect as a large amount data are produced online from the sensors and the actuators at the field level (Harjunkoski, 2015). Also the interoperability between industrial automation and information systems is a critical concern during the development of an intelligent and knowledge-aware platform. In general process systems produce each day a large amount of real-time data that provide significant information, which currently is analyzed using conventional tools (Muñoz, 2012) whereas the decision-making is partially performed at a later stage using respective tools by the plant operators and the process managers. Therefore, the motivation of this work is mainly the development of a knowledge-based ontology-assisted platform that will enable online decision support procedures using real-time data combined with previously derived information from the process operation. 


\section{Knowledge-enabled Supervisory Monitoring Platform - From the Automation System to the Data Repository}

The traditional process plants rely on automation architectures (such as Supervisory Control and Data Acquisition - SCADA) that monitor and control the subsystems by real-time measurements from the $\mathrm{I} / \mathrm{O}$ field. The analysis of the operation and the performance evaluation are conducted at a secondary stage using data that are stored at Process Information Management Systems (PIMS). The proposed Knowledge-enables Supervisory Monitoring (KSM) platform aims at enhancing the information that can be online to the operators by realizing a first level online evaluation of the process operation. Therefore, besides the collection, analysis and deployment of informative actions, the motivation for the development of an advanced knowledge-based platform is the need for smart data visualization combined with diagnosis and alarm notifications capabilities (Harjunkoski, 2015). In that context the real-time and engineering data of a process unit needs to be interpreted in the specific engineering domain in order to gain an extra layer of insight for the process operation. This interpretation results in a knowledge-enabled platform that can suggest informative actions to the operators using a service-oriented architecture. The proposed approach is tested in a continuous process environment, but it can be also adopted in the discrete manufacturing and batch manufacturing industries.

\subsection{Analysis of the Platform}

Initially the knowledge about the process plant and the automation system must be derived from the lists of equipment and schematics and should be stored in an appropriate format (data repository). Also the connection between equipment and operations is created. In that context the KSM platform consists of an Ontology, a Common Information Data Exchange Module (CIDEM) and the Rules of operation. Figure 1 shows the architecture of the KSM platform and the connection to the process unit.

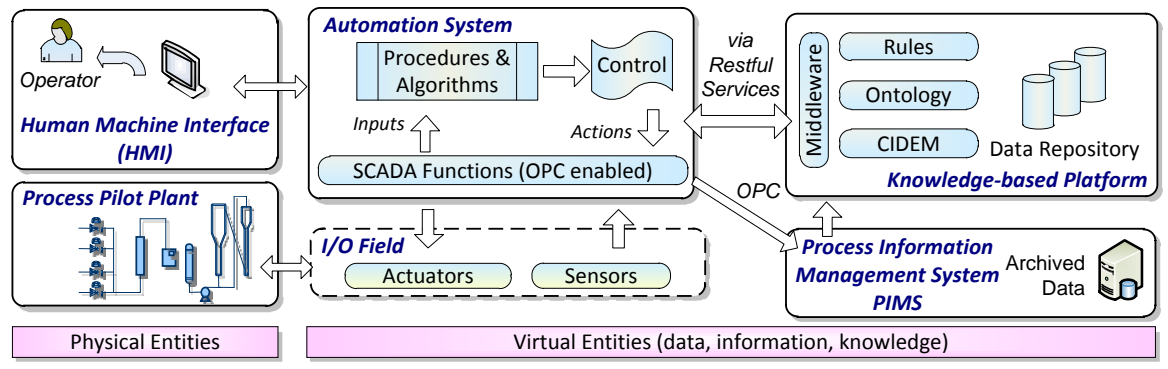

Figure 1 Architectural components and information flow of the SCADA/HMI and the Knowledge-based platform

The KSM platform analyzes the functions performed by the automation system of the process and also defines other functions typically associated with the process operations. The main areas supported by the platform are the data collection, the performance analysis, the events/alarms tracking and the process definition. Overall the scope of the platform is to provide interfaces that will allow the connection of all of the components of the automation system to the smart knowledge-aware functions (such as the local control algorithms, physical process equipment, and operation rules/conditions) and to define the formalism that supports heterogeneous information to be integrated and evaluated based on predefined methods. 
An intelligent decision making and notification system based on a knowledge-enabled supervisory monitoring platform

\subsection{Information exchange and data filtering}

The KSM platform is complemented by online processing filtering tools. These filtering tools are able to real-time combine the incoming event information with the historical data already stored in the repository extracting useful semantic information about them. The filtering tool is developed that uses online data from the SCADA system to invoke the specific rule of operation. Online data from the process and the information which is derived by the historical data are combined by the filtering tool. The bidirectional communication relies on an OPC-enabled middleware using Restful services whereas the visualization of the resulting information is integrated at the existing interfaces through the process database at the automation system. For example, an incoming measurement event, before it is stored, it is examined by the CIDEM against historical information trying to identify possible similarities with alerts and measurements that are connected to incidents. When a similarity with an abnormal event is identified, then a notification is sent back to the automation system and the operator is notified, so as to proceed with the necessary procedures in order to check this potential event.

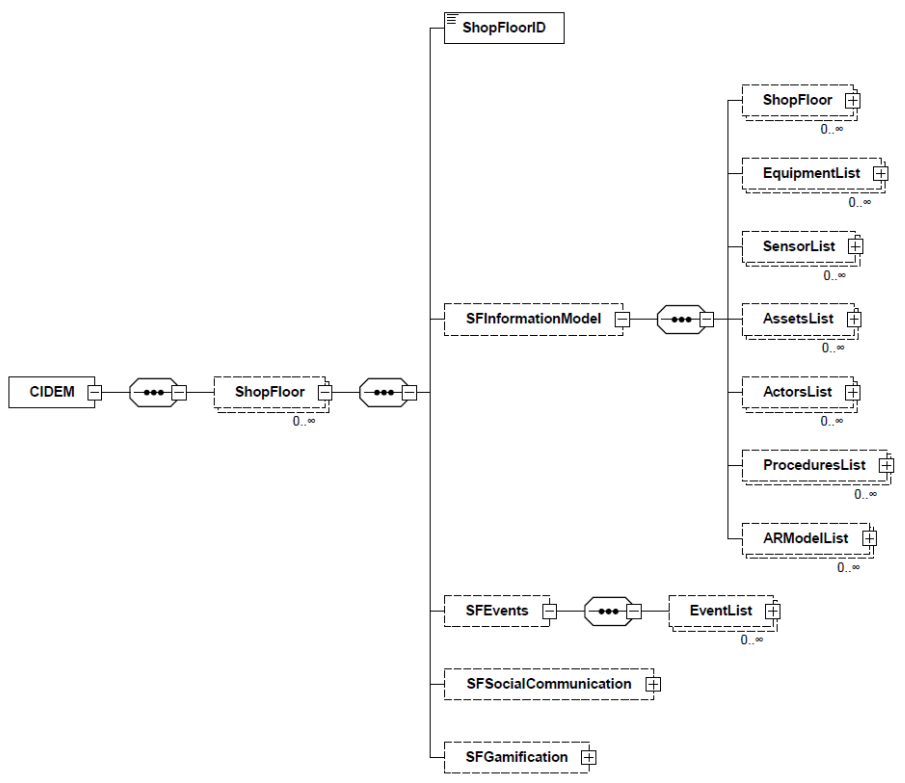

Figure 2: Developed ontology

\subsection{Rules of operation for behaviour extraction}

A Finite State Machine (FSM) formulation is used to describe the processes during which information or tasks move from one state to another for action, according to a set of rules (Ziogou, 2013). They can be fully semi-automatic or completely automatic, depending on the involvement of the process operator or not. The scope of the invoked rules is to extract information to notify the operator about the evaluation of the performance of the involve plant components and they can be modeled by a Finite state machine (FSM) which is a dynamic approach that describes the evolution in time of a set of discrete and continuous state variables. A FSM M is defined by a tuple $\left(Q, q_{0}, \delta, \lambda, \mathrm{X}, \mathrm{Y}\right)$ in which $\mathrm{Q}$ is a finite set of states, $q_{o} \in Q$ is the initial state, $\delta$ is the state transfer function, $\lambda$ is the output function, $X$ is the finite input alphabet; and $Y$ the 
finite output alphabet. The FSM represents all feasible states of the components and the logical rules that trigger the transitions between the states.

\section{Common Information Data Exchange Model}

The plant ontology is the knowledge representation of the plant structure, the hierarchical equipment structure and the equipment interconnections (Venkatasubramanian, 2006). Based on that a Common Information Data Exchange Model (CIDEM) has been developed, aiming to provide a model of information elements (e.g. concepts, events, relations, interfaces) used for information exchange. The CIDEM definition is considered as a shared vocabulary which is used to address the information needs of the plant-floor components. More specifically CIDEM defines how managed elements are represented as a common set of objects along with the relationships between them (DSP0201,2009). Standards such as B2MML (Business To Manufacturing Markup Language), which is an ISA-95 XML implementation has been utilized (ANSI/ISA-95.00.03-2005). The CIDEM specification consists of architecture and concepts, language (by which the CIDEM schema is defined), and a method for mapping CIDEM to other information models. The architecture is object-oriented, while its elements are represented as classes, whereas the relationships are represented as associations. The main elements of the CIDEM ontology are shown in Figure 2.

\subsection{Analysis of Information Stored at the CIDEM}

The CIDEM models both static (SFinformationModel) and dynamic data (SFevents) of the plant-floor. Based on this ontology, CIDEM is able to receive and store the static information of the plant-floor, which is comprised by the plant-floor geometry, the equipment (Id, description, type, location, etc.), the sensors (Id, description, type, location, etc.) that are used for monitoring the devices/equipment, as well as the assets, the actors and the procedures that describes the operations in the plant-floor environment.
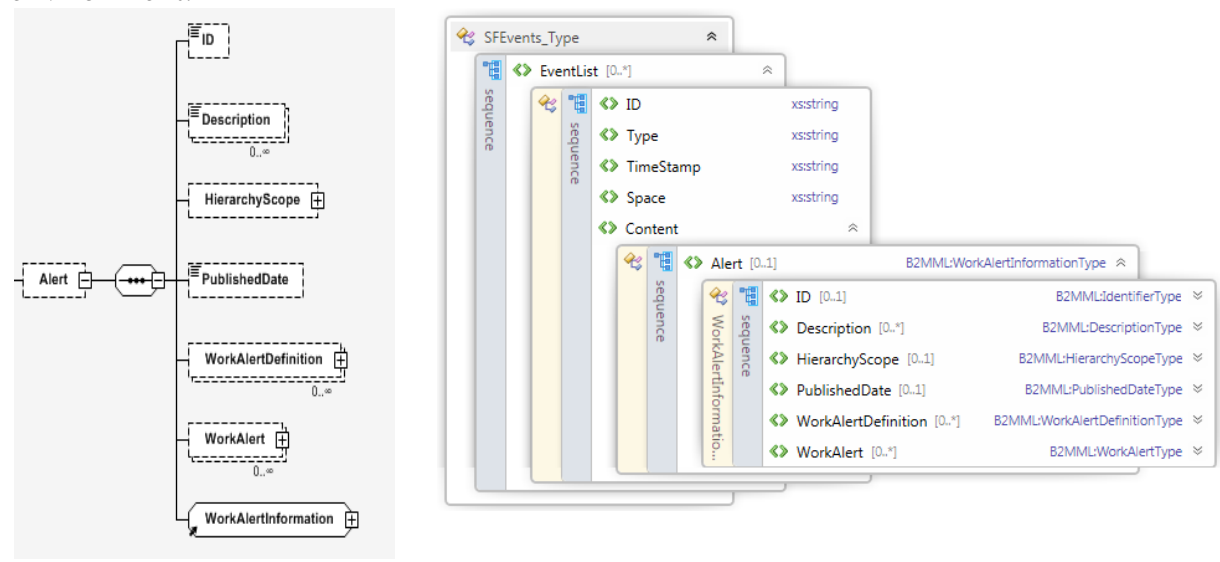

Figure 3: Alerts schema and properties

On the other hand, the dynamic information of the plant-floor is comprised of the events, which are divided into two main categories, the measurements and the alerts. Each event is characterized by an unique identification number, its type (measurements or alert), the timestamp that it is occurred, the space (location), the source that produce it and other information describing in more details the event according to ISA-95. Furthermore, the CIDEM is specified by the inputs/outputs interfaces between the 
An intelligent decision making and notification system based on a knowledge-enabled supervisory monitoring platform

interconnected components and the data repository. An indicative example of the XSD schema describing the alerts in the CIDEM is shown in Figure 3.

\section{CERTH's Use Case - Synthesis Gas Pilot Plant}

The demonstration of the developed platform will be instantiated at a pilot plant which is designed and operated at the premises of CERTH. This unit is a continuous process that has been used for steam reforming and partial oxidation of methane or ethane and aqueous mixtures of hydrocarbons such as methanol and bio-oil. The unit supports a spout bed type reactor and a fixed bed reactor used alternatively for preliminary activity measurements. Figure 4 shows the simplified process flow diagram (PFD) of the unit.

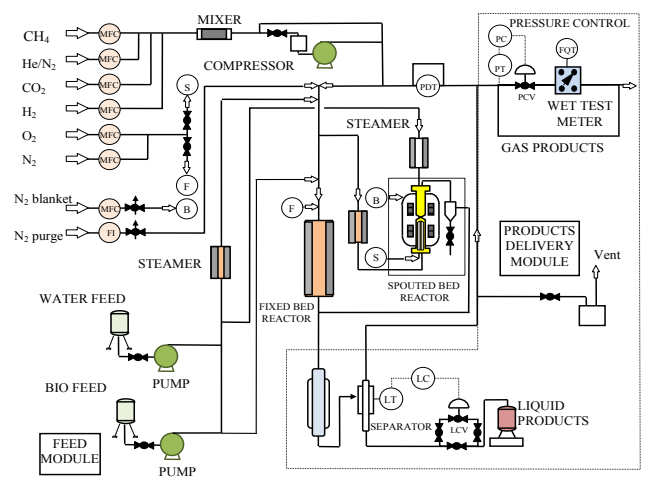

Figure 4 PFD of SynGas

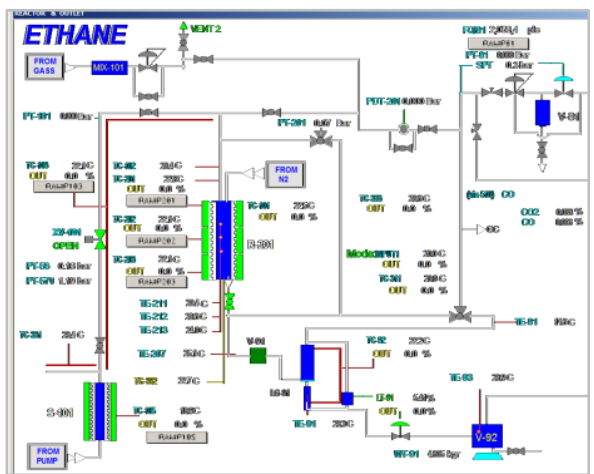

Figure 5 Indicative HMI of SynGas

The pilot plant is fully automated and intuitive HMIs represent the alternative flowsheet of either reactor. The automation system relies on a supervisory control and data acquisition (SCADA) architecture. All system components (pumps, heaters, valves and so forth) are controlled by on/off commands or by pre-programmed start-up procedures. The monitoring system has been developed in order to provide real-time information regarding the state of the plant, as well as, trending features related to the evolution of the experiments. Figure 5 shows and indicative interface which is used for the monitoring of the process status.

\subsection{Instantiation of the Supervisory Monitoring Platform}

The knowledge-enabled platform provides information related to early warnings of failing experiments or notification for preventive maintenance. An indicative case is the use of the evolution of the temperature profile, or the consumption of power by the heating resistance by observing the controller output besides the input. In the case of a significant deviation it is an indication related to a potential malfunction of the heating resistance or the electrical components related to the electric circuit.

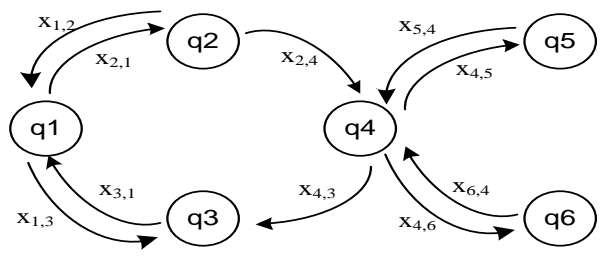

$$
\begin{aligned}
& q_{1}: \text { Standby mode } \\
& q_{2}: \text { Operation mode } \\
& q_{3}: \text { Fault or Disconnected } \\
& q_{4}: \text { Maintenance } \\
& q_{5}: \text { Performance degradation } \\
& q_{6}: \text { Abnormal response }
\end{aligned}
$$

Figure 6 FSM for a heating zone at the chemical reactor 
The CIDEM represents both static (e.g. data related to the reactor operating ranges) and dynamic data (e.g. the temperature of the reactor) of the pilot plant combined with information from the Process and Instrumentation Diagram (P\&ID) of the plant. The KSM platform provides notification based on a set of predefined rules which are modeled by a FSM structure. Figure 6 shows an indicative example for the behavior analysis of the components related to a heat-up zone of a chemical reactor, which are a temperature sensor and a heating resistance (actuator).

The states $\left(q_{1}, . . q_{6}\right)$ describe the possible conditions of a heating zone during all potential modes of operation of the process (startup/shutdown, running experiment, maintenance, preparation for operation). The transition between the states is performed according to a set of rules that define the input alphabet $(X)$. The notification to the user is performed only when state $q_{5}$ and $q_{6}$ are reached. At these states the historical data of the behavior of the sensor and the actuator are evaluated at regular intervals against the real-time data. The evaluation can activate the respective transition when the value of the controller output is beyond the expected average or if there is a significant change with an increasing slope of the output. In order for the state to change the user response (acknowledgement of notification) is necessary for states $q_{5}$ and $q_{6}$.

\section{Conclusions}

The main results of this work is the development of a knowledge-based system that can enhance the information which is made available to the process operator in an integrated not disruptive way combining both online and historical data from the $\mathrm{I} / \mathrm{O}$ field and the previously applied actions. One of the future extensions will be to include data from both the smart sensor network and the movement and thermal detection sensors in order to derive information that will include operator's behaviour and status of equipment that will not rely only at the existing measurements of the I/O field, such as detection of an abnormal movement within a close proximity to the pilot plant.

\section{Acknowledgment}

The authors acknowledge the support of EC project: 'SATIFACTORY - A collaborative and augmented-enabled ecosystem for increasing satisfaction and working experience in smart factory environments'.

\section{References}

ANSI/ISA-95.00.03-2005, 2005, Enterprise-Control System Integration. Part 3: Activity models of manufacturing operations management, ResearchTriangle Park,NC: ISA

DSP0201, 2009, Representation of CIM using XML, Distributed Management Task Force, Inc., Version: 2.3.1

I. Harjunkoski, 2015, Industrial perspectives on the deployment of scheduling solutions, Computer Aided Chemical Engineering, 37, 63-70

E. Muñoz, E. Capón-García, A. Espuña, L. Puigjaner, 2012, Ontological framework for enterprise-wide integrated decision-making at operational level, Computers \& Chemical Engineering, 42, 217-234

V. Venkatasubramanian, C., Zhao, G., Joglekar, A., Jain, L., Hailemariam, P., Suresh, P., et al., 2006, Ontological informatics infrastructure for pharmaceutical product development and manufacturing, Computers \& Chemical Engineering, 30, 1482-1496.

C. Ziogou, D. Ipsakis, P. Seferlis, S. Bezergianni, S. Papadopoulou, S. Voutetakis S, 2013, Optimal production of renewable hydrogen based on an efficient energy management strategy, Energy, 55, 58-67 